\title{
Managing COVID-19 related distress in primary care: principles of assessment and management
}

\author{
Laurence Astill Wright ${ }^{*}$ (D), Sam Gnanapragasam², Anthony J. Downes ${ }^{1}$ and Jonathan I. Bisson ${ }^{1}$
}

\begin{abstract}
COVID-19 will cause normal feelings of worry and stress and many of those who experience higher levels of distress will experience resolution of their symptoms as society returns to pre-COVID-19 functioning. Only a minority are likely to develop a psychiatric disorder. Certain individuals may be vulnerable to experiencing persisting symptoms, such as those with pre-existing comorbidity. Management approaches could centre around using collaborative approaches to provide and build on already existing socioeconomic support structures, the avoidance of over-medicalisation, watchful waiting and finally treating those who do meet the criteria for psychiatric diagnosis. Primary care clinicians are likely be the first healthcare point of contact for most COVID-19 related distress and it is important that they are able to provide evidence based and evidence informed responses, which includes social, psychological and pharmacological approaches. This expert opinion paper serves to summarise some approaches, based primarily on indirect extrapolation of evidence concerning the general management of psychological distress, in the absence of COVID-19 specific evidence, to assist primary care clinicians in their assessment and management of COVID-19 related distress.
\end{abstract}

Keywords: Psychological Distress, Traumatic Stress Symptoms, Anxiety, Depression, PTSD, COVID-19

The psychological consequences of COVID-19 are likely to be broad and affect millions of people worldwide. COVID-19 may cause psychological and emotional distress, commonly manifesting in grief and in symptoms of anxiety, depression and traumatic stress, much of which will represent a normal reaction to an abnormal situation and subside without the need for formal intervention. Primary care clinicians are likely to be the first healthcare point of contact for most COVID-19 related distress and it is important that they are able to provide evidenceinformed responses, which includes social, psychological and pharmacological approaches. This paper, which is based on a non-systematic review of the literature and

\footnotetext{
*Correspondence: laurencewright@doctors.org.uk

${ }^{1}$ Division of Psychological Medicine and Clinical Neurosciences, Cardiff

University School of Medicine, Cardiff, UK

Full list of author information is available at the end of the article
}

expert opinion, aims to explore and predict patterns of psychological distress during the COVID-19 pandemic, including when and what interventions to offer in primary care.

\section{Stressors, manifestations and course of distress}

The trajectory of COVID-19 and magnitude of its impact on life across the world, suggest that the psychological challenges may differ from previous pandemics in scale and possibly also in nature via multiple psychosocial stressors and secondary socioeconomic consequences. COVID-19 may affect individuals in a variety of ways with direct effects on individuals (e.g., via hospitalisation and bereavement) and indirect effects (e.g., social isolation, financial hardship), which are compounded by shifting government policies (Fig. 1). As such, it is likely that COVID-19 will have a profound impact on the social determinants of health, including disruptions 


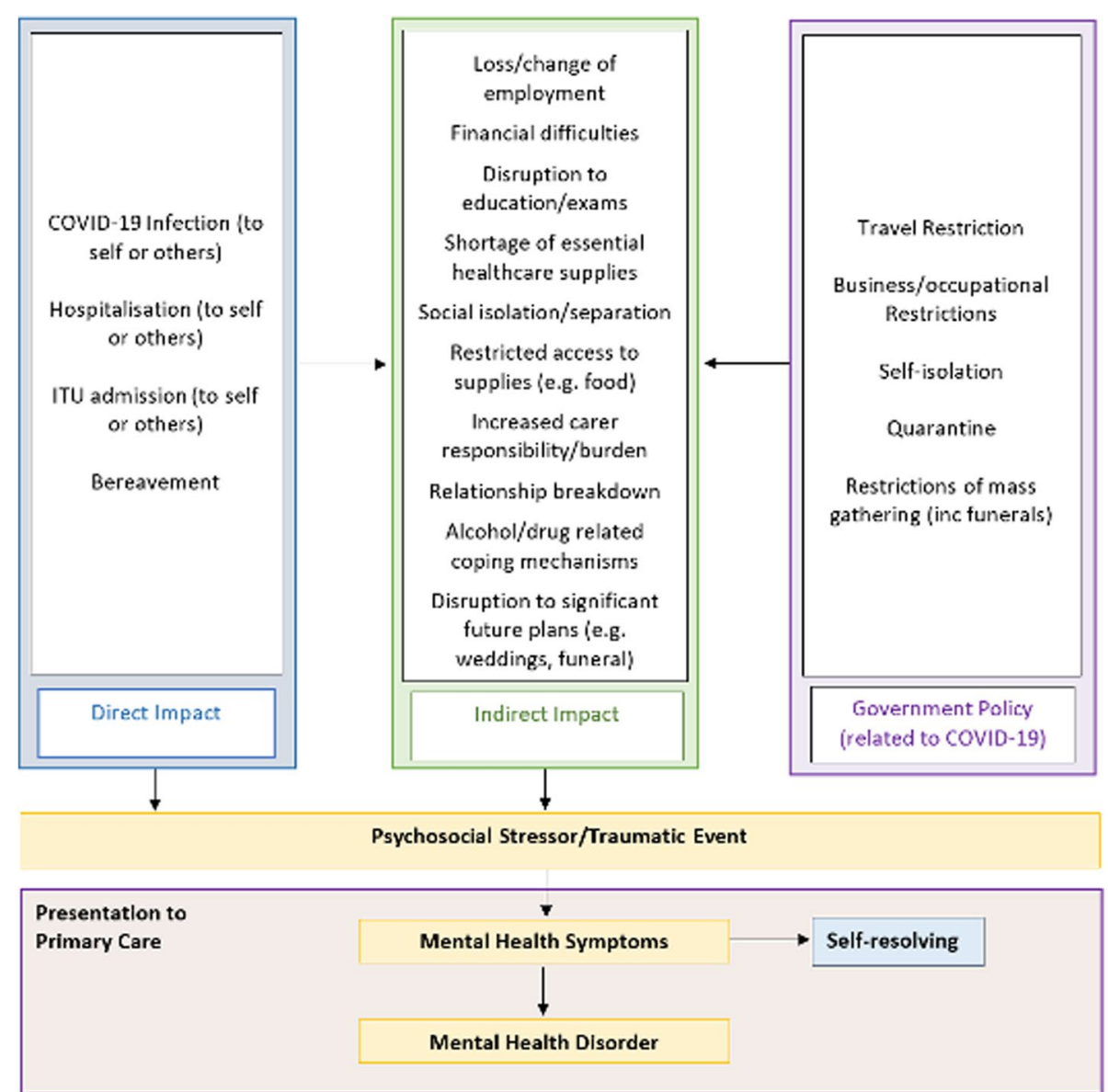

Fig. 1 COVID-19 related direct and indirect causes and symptoms of psychological distress

to healthcare provision, education, economic stability, community networks and accessibility to physical environment.

Some of these direct life events will meet the traumatic stressor criterion for Diagnostic and Statistical Manual of Mental Disorders Fifth Edition (DSM-5) Post traumatic Stress Disorder (PTSD), such as experiencing or witnessing actual or threatened death. However, many indirect stressors will not lead to PTSD but might still cause distress. Stressful life events are one of the main precipitating factors for psychopathology and COVID-19 related psychosocial stressors will likely precipitate a range of psychological reactions, including mental disorder.

It is common and normal for individuals to feel stressed during a time of such significant global upheaval (Fig. 1). For most people, this initial psychological upset will gradually decrease as the external stressor subsides. The majority of the general population will show good adaptability and resilience to psychosocial stressors, and some individuals may even have positive experiences (e.g. pride in their ability to cope in crisis ([1] and reduced anxiety amongst some adolescents [2]). Only a minority of individuals are likely to experience pathological distress which will probably be characterised as symptoms of anxiety, depression, grief, traumatic stress and maladaptive coping behaviours such as substance misuse [3].

These predictions are supported by the emerging evidence. Recent systematic reviews and meta-analyses demonstrate wide ranging prevalence rates of symptoms of anxiety, depression and traumatic stress [4-6]. Peer reviewed studies of higher methodological quality demonstrated lower prevalence rates of posttraumatic stress (17.38\% in published studies) compared with those of lower methodological quality $(34.71 \%$ in unpublished studies) [4]. In the United Kingdom, a study of 2000 adults representative of the British population 52 days after the first confirmed case of COVID-19 demonstrated only slightly higher rates of traumatic stress (female: $14.9 \%$, male: $18.9 \%$ ), anxiety (female: $25.1 \%$, male $17.9 \%$ ) and depression (female: $23.4 \%$, male $20.6 \%$ ) compared to pre-COVID-19 UK population estimates [3]. This highlights the remarkable psychosocial resilience of the 
majority of the general population. While direct comparisons are not possible due to methodological differences, these findings are broadly similar to the prevalence of distress experienced during SARS-CoV-1 [7, 8].

Studies examining the psychological consequences of other pandemics [9] suggest that some non-specific symptoms of emotional distress (which have been characterised as adjustment disorder-psychological distress in response to a significant life event - by some authors) will be just as common as those of PTSD [10]. COVID19 related mortality will also leave many people bereaved, experiencing entirely normal but highly distressing symptoms related to the anguish of losing someone close, such as anger, guilt, regret and loneliness. Furthermore, the cultural norms that support a normal grieving process will be disrupted (e.g., inability to attend their funeral), increasing the likelihood of pathological outcomes such as prolonged grief disorder. Loss of social support due to bereavement or unemployment, could add to the risk of individuals developing psychiatric problems and maladaptive coping strategies such as substance abuse.

Similar to normal feelings of worry and stress related to COVID-19, many of those who experience higher levels of distress will experience resolution of their symptoms as society gradually returns to pre-COVID-19 functioning. Only a minority may develop a psychiatric disorder, such as anxiety, depression, adjustment disorder, prolonged grief disorder and PTSD [11]. Relatively few studies have assessed the rate of formal psychiatric disorder in the general population following epidemics, with most using assessment measures insufficient to diagnose [12] and not assessing pre-existing psychiatric comorbidity [11].

\section{At risk population groups}

Prevalence studies of hospitalised SARS patients have demonstrated a psychiatric disorder prevalence of $42.5 \%$ in 233 individuals up to 4 years post-infection, albeit in a particularly high risk group [13]. Those requiring higher levels of medical input for COVID-19 related illness will be more likely to develop psychiatric conditions requiring treatment, as observed following the 2009 H1N1 pandemic [14]. Estimates of total numbers of patients requiring critical care vary markedly, but of confirmed cases of COVID-19, however, 15\% will require hospitalisation and $5 \%$ will require invasive mechanical ventilation on an Intensive Therapy Unit [15]. The prevalence of PTSD symptoms following ITU discharge is $24 \%$ at 6 months and $22 \%$ at 12 months [16, 17], comparable to that of military combat and major physical injury [18]. Analysis of patients treated for SARS-CoV-1 highlights that greater perceived life threats are associated with worse PTSD symptom severity [19]. This imminent threat to life by an entirely novel and poorly understood virus may be combined with worry of spreading the contagious virus to vulnerable friends and family.

COVID-19 related distress will likely disproportionally affect those with more traumatic COVID-19 exposure and other more vulnerable groups, including those with pre-existing psychiatric and physical health comorbidity [3]. As most people with pre-existing comorbidities are managed within community settings this will pose particular challenges to primary care services. Multiple shared vulnerability factors, such as adverse childhood experiences and low socioeconomic status, are common trans-diagnostically with pre-existing psychiatric disorder likely to increase susceptibility to other psychiatric conditions [20]. Furthermore, COVID-19 may exacerbate pre-existing socioeconomic vulnerability and economic recessions are associated with increased prevalence of psychiatric morbidity [21].

Healthcare workers who are predominantly female, may be particularly vulnerable [22], with healthcare workers with SARS demonstrating poor emotional adjustment following physical illness [12]. Clear communication and support within the workplace clearly affects subsequent distress, [23] and while individuals may not initially appreciate the severity of their distressing experiences at the time, employers should consider the mental health consequences of inadequate support structures [24]. Other occupations with high viral exposure requiring institutional support include supermarket workers.

Early evidence suggests that there is a higher COVID19 related psychological distress prevalence in women, who are also more likely to be bereaved [25, 26]. A multitude of social and biological differences explain the higher rates of PTSD in women regardless of trauma type [27]. Women tend to seek more social support than men, and isolation and quarantine will decrease this available social support, a lack of which is the most consistent correlate of poor outcomes after trauma [27]. Early reports of increased call volumes to the National Domestic Abuse Helpline [28] further highlight women's potential vulnerability.

Lack of social support is likely to exacerbate distress related with both COVID-19-related and non-COVID19-related traumatic events. While isolation and quarantine does not meet the PTSD stressor criterion, some individuals may feel the experience overwhelms their psychological ability to cope. The psychological impacts of quarantine and isolation are broad and highlight the effect of isolation greater than 10 days in increasing traumatic stress symptoms, anger and avoidance [12]. Interestingly, early research suggests that people over 65 report lower rates of COVID-19 related anxiety and depression compared to younger people, suggesting 
greater psychosocial resilience despite higher risk of serious illness [3, 29].

\section{Principles of intervention \& treatment approaches}

There remains considerable uncertainty in how best to manage the psychosocial consequences of COVID-19. This paper summarises the approaches recommended by a variety of organisations $[1,30]$, based primarily on the indirect extrapolation of evidence concerning the general management of psychological distress, in the absence of COVID-19 specific evidence. The large heterogeneity in demographics affected by COVID-19 will cause varied manifestations of psychological distress requiring a range of approaches (Fig. 2). General overarching principles are centred around the avoidance of over-medicalisation and further harm, using collaborative approaches to provide and build on already existing socioeconomic support structures, watchful waiting and finally treating those who do meet the criteria for psychiatric diagnosis [1]. Stepped care interventions should be multi-faceted and function within an integrated support system of multiple coordinating groups acting in response to COVID-19 [1].

Initially, individuals will require easy access to basic services providing practical and financial security to protect their health and wellbeing. These services should be safe, socially appropriate, preserve the dignity of those accessing them and be able to adapt to changing needs [1], e.g. ensuring vulnerable groups still retain access to shelter, care and medication. Following this, the COVID-19 response should seek to enable and extend existing family and community support structures through providing COVID-19 specific knowledge, teaching new supportive skills (such as psychological first aid) and increasing awareness of the care pathways in place for distressed individuals to access specialist support [1]. Employers of staff vulnerable to psychological distress should implement clear guidance around policies, practices and occupational support structures [31] and avoid stigmatising those with COVID-19 or related distress [30].

For those individuals displaying heightened distress, primary care practitioners can offer emotional, practical and pragmatic support. This serves to cope with worries arising directly from COVID-19 (e.g., exposure to the disease and survivors' stories of suffering, loneliness and powerlessness) and those indirectly related (e.g., an inability to engage in recreation, poor organisation and occupational role definitions) [31]. Individuals should be encouraged to maintain the social support structures and coping strategies they already possess within the confines of social distancing [30] but there is no one size fits all solution to the psychosocial requirements of an entire society [1]. Coping strategies may include selfcare techniques, such as relaxation and cognitive exercises, physical exercise and providing support on how to access reliable information about COVID-19 related developments.

\section{COVID-19 related mental illness}

The ability to distinguish between a normal reaction to external stressors and a pathological one will be key for primary care practitioners, who may already struggle to differentiate between psychological distress and mental

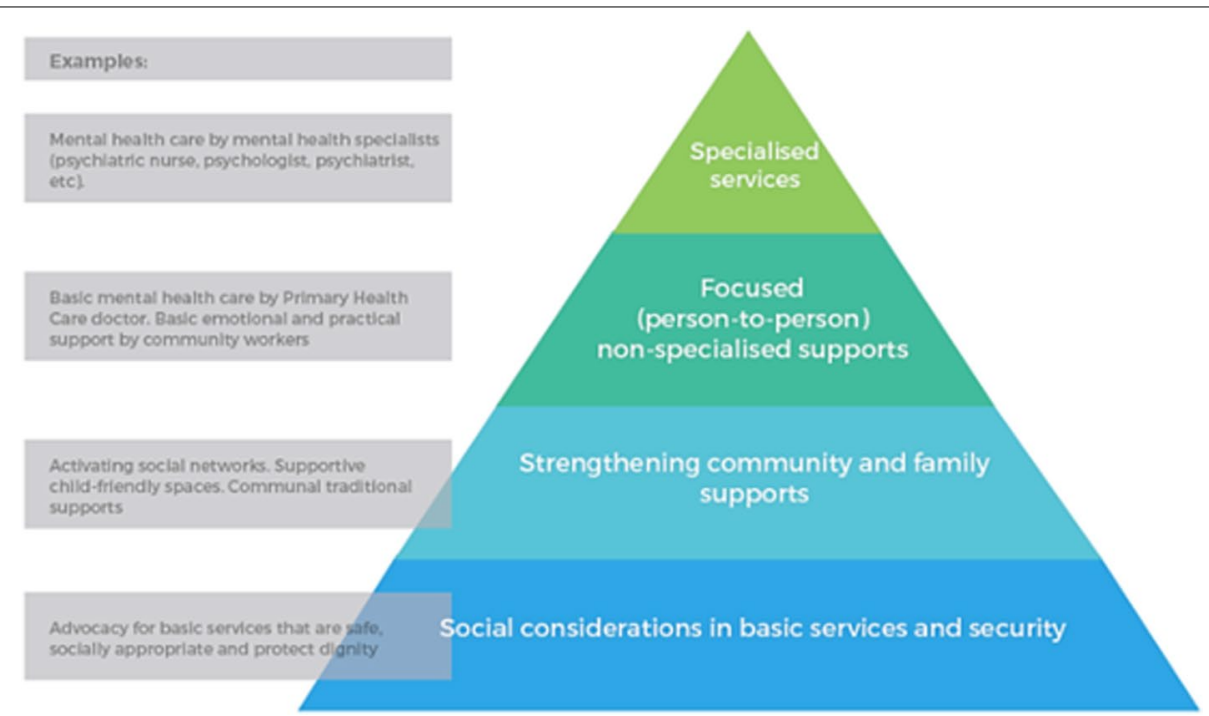

Fig. 2 Intervention pyramid for mental health and psychosocial support (Reproduced from IASC 2020_permission has been requested to reproduce this figure) 
disorder due to contrasting frameworks around the conceptualisation of each [32]. This may be particularly challenging acutely, before the possibility of longitudinal assessment and before any basic supportive interventions have been offered [33]. Pathological reactions typically involve a significant impact on an individual's functioning with a higher severity of symptoms persisting over a prolonged period of time and often beyond the resolution of a precipitating psychosocial stressor. Pathological reactions may be associated with higher degrees of risk, the management of which should be a key consideration during initial consultation. Assessment should appreciate that while direct COVID-19 stressors may resolve first, for many, the indirect impact of the pandemic, such as financial hardship, will persist for longer.

For the small minority of individuals who develop signs and symptoms of mental disorder and for whom basic emotional and pragmatic support is insufficient, evidence-based psychological and/or pharmacological treatments should be offered based on the primary presenting diagnosis (e.g. Depression [33], PTSD [34]). Many of these interventions will be provided in primary care settings but some individuals will have more complex difficulties or risks, including suicide risk, that require involvement of secondary care services.

\section{Limitations}

This paper, which is not the result of a systematic literature search, is based on a non-systematic review of the literature and our knowledge of the area, serves to summarise some of the available evidence to assist primary care clinicians in their assessment and management of COVID-19 related distress. Consistent with a recently published rapid systematic review of the literature [35], most of the studies considered have limited internal and external validity and a high risk of selection bias (small sample sizes from single geographical regions with short follow up periods). Consequently, our recommendations should be employed judiciously and always in a personalised manner that integrates clinical expertise, individual patients' values and preferences, and the available evidence.

\section{Conclusions}

COVID-19 related distress may manifest in symptoms of depression, anxiety, grief and traumatic stress, the majority of which does not require formal medical treatment. Those particularly vulnerable to persisting symptoms and the development of psychiatric conditions are those with pre-existing conditions, females, health care workers, those with low social support, possibly exacerbated by isolation and quarantine, and those facing indirect socioeconomic consequences such as unemployment. For those who develop mental health disorders, effective evidence-based treatments are available. The psychiatric consequences of the COVID-19 pandemic will likely first present in primary care and primary care clinicians have the potential to mitigate their consequences.

\section{Acknowledgements \\ Not applicable}

\section{Patient and public involvement}

This research was done without patient involvement. Patients were not invited to comment on the study design and were not consulted to develop patient relevant outcomes or interpret the results. Patients were not invited to contribute to the writing or editing of this document for readability or accuracy.

\section{Authors' contributions}

This article was conceptualised by LAW, SG and JB. The original draft was led by LAW. The manuscript was reviewed and edited by LAW, SG, JB, and AD prior to submission. The authors read and approved the final manuscript.

\section{Funding}

This work was supported by a Wellcome Trust ISSF3 Clinical Primer to LAW and an NIHR Academic Clinical Fellowship to SG. The funding body had no influence on the design of the study, the collection, analysis, and interpretation of data and in writing the manuscript.

Availability of data and materials

Not applicable.

\section{Declarations}

\section{Ethics approval and consent to participate}

Ethical approval was not required for this study.

\section{Consent for publication}

Not applicable.

\section{Competing interests}

JB: Awards of grant funding to support research from various sources to conduct research in the field of traumatic stress, including the National Institute for Health Research, Forces in Mind Trust and Medical Association for Psychedelic Studies; and development of a guided self help intervention owned by Cardiff University for which he could receive a share of royalties in the future.

\section{Author details}

${ }^{1}$ Division of Psychological Medicine and Clinical Neurosciences, Cardiff University School of Medicine, Cardiff, UK. ${ }^{2}$ The Institute of Psychiatry, Psychology and Neuroscience, King's College London, London, UK.

Received: 11 June 2020 Accepted: 23 February 2021

Published online: 14 April 2021

\section{References}

1. IASC (Inter Agency Standing Committee). Addressing mental health and psychological aspects of COVID-19 outbreak. 2020. Accessed 5 Apr 2020. Available at: https://interagencystandingcommittee.org/iasc-referencegroup-mental-health-and-psychosocial-support-emergency-settings/ interim-briefing

2. Widnall, E. Winstone, L. Mars, B. Haworth, C. Kidger, J. Young people's mental health during the COVID-19 pandemic: initial findings from a secondary school survey study in South West England. NIHR School for Public Health Research. 2020.

3. C19PRC (COVID-19 Psychological Research Consortium). Initial research findings on COVID-19 and mental health in the UK. 2020. Accessed 5 Apr 
2020. Available at: https://drive.google.com/file/d/1A95KvikwK32ZAX 387nGPNBCnoFktdumm/view

4. Cooke J, Eirich R, Racine N, Madigan S. Prevalence of posttraumatic and general psychological stress during COVID-19: A rapid review and metaanalysis. Psychiatry Res. 2020;292:113347. https://doi.org/10.1016/j.psych res.2020.113347.

5. Xiong J, Lipsitz O, Nasri F, Lui L, Gill H, Phan L, Chen-Li D, lacobucci M, Ho R, Majeed A, McIntyre R. Impact of COVID-19 pandemic on mental health in the general population: A systematic review. J Affect Disord. 2020;277:55-64. https://doi.org/10.1016/j.jad.2020.08.001.

6. Salari N, Hosseinian-Far A, Jalali R, Vaisi-Raygani A, Rasoulpoor S, Mohammadi M, Rasoulpoor S, Khaledi-Paveh B. Prevalence of stress, anxiety, depression among the general population during the COVID-19 pandemic: a systematic review and meta-analysis. Glob Health. 2020;16(1):111. https://doi.org/10.1186/s12992-020-00589-w.

7. Wu, K. Chan, S. Ma, T. Posttraumatic Stress after SARS. Emerg Infect Dis. 2005;11(8). https://doi.org/10.3201/eid1108.041083

8. Wu, K. Chan, S. Ma, T. Posttraumatic stress, anxiety, and depression in survivors of severe acute respiratory syndrome (SARS). J Trauma Stress. 2005:18(1). https://doi.org/10.1002/jts.20004

9. Rogers J, Chesney E, Oliver D, Pollak T, McGuire P, Fusar-Poli P, Zandi MS, Lewis G, David A. Psychiatric and neuropsychiatric presentations associated with severe coronavirus infections: a systematic review and metaanalysis with comparison to the COVID-19 pandemic. Lancet Psychiatry. 2020;7(7):611-27. https://doi.org/10.1016/S2215-0366(20)30203-0.

10. Liu J, Bao Y, Huang X, Shi J, Lu L. Mental health considerations for children quarantined because of COVID-19. Lancet Child Adolesc Health. 2020. https://doi.org/10.1016/S2352-4642(20)30096-1.

11. Gardner, P \& Moallef, P. Psychological impact on SARS survivors: critical review of the English language literature. Can Psychol. 2015;56(1). https:// doi.org/10.1037/a0037973

12. Brooks, S. Webster, R. Smith, L. Woodland, L. Wessely, S. Greenberg, N. Rubin, G. The psychological impact of quarantine and how to reduce it: rapid review of the evidence. Lancet. 2020;395(10227). https://doi.org/10. 1016/S0140-6736(20)30460-8

13. Lam, M. Wing, Y. Yu, M. Leung, C. Ma, R. Kong, A. So, W. Fong, S. Lam, $S$. Mental morbidities and chronic fatigue in severe acute respiratory syndrome survivors: long-term follow-up. Arch Intern Med. 2009;169(22). https://doi.org/10.1001/archinternmed.2009.384

14. Luyt, C. Combes, A. Becquemin, M. Beigelman-Aubry, C. Hatem, S. Brun, A. Zraik, N. Carrat, D. Grenier, P. Richard, J. Mercat, A. Brochard, L. Brun-Buisson, C. Chastre, J. REVA Study Group. Long-term outcomes of pandemic 2009 influenza A(H1N1)-associated severe ARDS. Chest. 2012;142(3). https://doi.org/10.1378/chest.11-2196

15. Rello J, Tejada S, Userovici C, Arvaniti K, Pugin J, Waterer G. Coronavirus disease 2019 (COVID-19): a critical care perspective beyond China. Anaesth Crit Care Pain Med. 2020. https://doi.org/10.1016/j.accpm. 2020.03.00

16. Parker, A. Sricharoenchai, T. Raparla, S. Schneck, K. Bienvenu, O. Needham, D. Posttraumatic stress disorder in critical illness survivors: a metaanalysis. Crit Care Med. 2015;43(5). https://doi.org/10.1097/CCM.0000000000 000882

17. Patel, M. Jackson, J. Morandi, A. Girard, T. Hughes, C. Thompson, J. et al. Incidence and risk factors for intensive care unit-related post-traumatic stress disorder in veterans and civilians. Am J Respir Crit Care Med. 2016;193(12). https://doi.org/10.1164/rccm.201506-11580C

18. Hoge, C. Castro, C. Messer, S. McGurk, D. Cotting, D. Koffman, R. 2004. Combat duty in Iraq and Afghanistan, mental health problems, and barriers to care. N Engl J Med. 2004;351(1). https://doi.org/10.1056/NEJMo a040603

19. Lee, A. Wong, J. McAlonan, G. Cheung, V. Cheung, C. Sham, P. et al. Stress and psychological distress among SARS survivors 1 year after the outbreak. Can J Psychiatry. 2007;52(4). https://doi.org/10.1177/0706743707 05200405

20. Breslau N, Davis GC, Andreski P, Peterson E. Traumatic Events and Posttraumatic Stress Disorder in an Urban Population of Young Adults. Arch
Gen Psychiatry. 1991;48(3):216-22. https://doi.org/10.1001/archpsyc. 1991.01810270028003

21. Frasquilho, D. Matos, M. Salonna, F. Guerreiro, D. Storti, C. Gaspar, T. Caldas-de-Almeida, J. Mental health outcomes in times of economic recession: a systematic literature review. BMC Public Health. 2016;16(115). https://doi.org/10.1186/s12889-016-2720-y

22. L Rains S Johnson P Barnett T Steare J Needle S Carr. et al. Early impacts of the COVID-19 pandemic on mental health care and on people with mental health conditions: framework synthesis of international experiences and responses Soc Psychiatry Psychiatr Epidemiol. 2020:1-12. https://doi. org/10.1007/s00127-020-01924-7

23. Chan, A. Huak, C. Psychological impact of the 2003 severe acute respiratory syndrome outbreak on health care workers in a medium size regional general hospital in Singapore. Occup Med. 2004;54(3). https:// doi.org/10.1093/occmed/kqh027

24. Huremovic, D. Psychiatry of pandemics: a mental health response to infection outbreak. 2019. ISBN: 9783030153458.

25. W Guan Z Ni Y Hu W Liang C Ou JC He. Characteristics of Coronavirus Disease, 2019 in China N Engl J Med. 2020 https://doi.org/10.1056/NEJMo a2002032

26. Pierce M, Hope H, Ford T, Hatch S, Hotopf M, John A, Kontopantelis E, Webb R, Wessely S, McManus S, Abel K. Mental health before and during the COVID-19 pandemic: a longitudinal probability sample survey of the UK population. Lancet Psychiatry. 2020;7(10):883-92. https://doi.org/10. 1016/S2215-0366(20)30308-4.

27. Olff, M. Sex and gender differences in post-traumatic stress disorder: an update. 2017. Eur J Psychotraumatol. 2017;8(4). https://doi.org/10.1080/ 20008198.2017.1351204

28. Refuge. $25 \%$ increase in calls to National Domestic Abuse Helpline since lockdown measures began. 2020. Accessed 14 Apr 2020. Available at: https://www.refuge.org.uk/25-increase-in-calls-to-national-domesticabuse-helpline-since-lockdown-measures-began/

29. P García-Portilla de la L Fuente Tomás TJ Bobes-Bascarán LZ Treviño PS Madera MM Álvarez IG Miranda LS Álvarez PA Martínez J Bobes. Are older adults also at higher psychological risk from COVID-19? Aging Ment Health. 2020:1-8. https://doi.org/10.1080/13607863.2020.1805723

30. WHO (World Health Organisation). Mental health and psychosocial considerations during the COVID-19 outbreak. 2020. Accessed: 05/05/2020. Available at: https://www.who.int/docs/default-source/coronaviruse/ mental-health-considerations.pdf

31. Williams, R. Murray, E. Neal, A. Kemp, V. Top ten messages for supporting healthcare staff during the COVID-19 pandemic. 2020.

32. Geraghty, A. Santer, M. Beavis, C. Williams, S. Kendrick, T. Terluin, B. Little, P. Moore, M. 2019.'I mean what is depression?'A qualitative exploration of UK general practitioners' perceptions of distinctions between emotional distress and depressive disorder. BMJ Open. 9(12). https://doi.org/10. 1136/bmjopen-2019-032644

33. NICE (National Institute for Health and Care Excellence). Depression in Adults: recognition and management - NICE guideline CG90. 2009. Accessed 12/4/20. Available at: https://www.nice.org.uk/guidance/cg90

34. Bisson J, Baker A, Dekker W, Hoskins M. Evidence-based prescribing for post-traumatic stress disorder. Br J Psychiatry. 2020;216(3):125-6. https:// doi.org/10.1192/bjp.2020.40.

35. Zimmerman, M. Ali, S. Jones, N. Maskrey, N. Practical tips for clinicians helping patients with COVID-related anxiety/distress. Oxford University Centre for Evidence-Based Medicine. 2020. Available at: https://www. cebm.net/covid-19/practical-tips-for-clinicians-helping-patients-withcovid-related-anxiety-distress/. Accessed 11/9/20.

\section{Publisher's Note}

Springer Nature remains neutral with regard to jurisdictional claims in published maps and institutional affiliations. 